\title{
Interval-Valued Complex Fuzzy Logic
}

\author{
Sarah Greenfield \\ Centre for Computational Intelligence \\ and DMU Interdisciplinary Group in \\ Intelligent Transport Systems (DIGITS) \\ School of Computer Science \\ and Informatics \\ De Montfort University \\ Leicester LE1 9BH, UK. \\ Email: s.greenfield@dmu.ac.uk
}

\author{
Francisco Chiclana \\ Centre for Computational Intelligence \\ School of Computer Science \\ and Informatics \\ De Montfort University \\ Leicester LE1 9BH, UK. \\ Email: chiclana@dmu.ac.uk
}

\author{
Scott Dick \\ Department of Electrical \\ and Computer Engineering \\ University of Alberta \\ Edmonton \\ Alberta, Canada. \\ Email: sdick@ualberta.ca
}

\begin{abstract}
Data is frequently characterised by both uncertainty and seasonality. Type-2 fuzzy sets are an extension of type-1 fuzzy sets offering a conceptual scheme within which the effects of uncertainties in fuzzy inferencing may be modelled and minimised. Complex fuzzy sets are type-1 fuzzy sets extended by an additional phase term which permits them to intuitively represent the seasonal aspect of fuzziness in time-series applications. Type$\mathbf{2}$ fuzzy sets take two forms, generalised, and the simpler interval. Interval-valued fuzzy sets are type-1 fuzzy sets whose behaviour and properties are equivalent to interval type-2 fuzzy sets. This paper is concerned with the combination of interval-valued fuzzy sets and complex fuzzy sets to develop interval-valued complex fuzzy sets, an adaption of complex fuzzy sets such that the membership function assigns each point on the domain to an interval.
\end{abstract}

From the definition of the interval-valued complex fuzzy set, the principles of interval-valued complex fuzzy logic are developed.

\section{INTRODUCTION}

It is 50 years since Zadeh [38] introduced the concept of a type-1 fuzzy set. In 1975, he went on to define the type-2 fuzzy set [39], [40], [41]. Complex fuzzy sets, applicable to the modelling of periodic phenomena, are a relatively recent development in fuzzy set theory, proposed by Ramot et al. in 2002 [30]. A natural extension of real-valued, or type-1 fuzzy sets, they differ from them in so far as their membership grades are complex numbers (of modulus $\leq 1$ ) [8]. A striking feature that type-2 fuzzy sets and complex fuzzy sets have in common is that they are three-dimensional.

In recent years, 'big data' has become a growing research concern. Data, whether big or otherwise, is frequently characterised by both uncertainty and seasonality. Uncertainty in the data may derive from factors such as corruption, or from elements being missing. Seasonality, whereby the data has an underlying repeating, cyclical pattern, is a very common characteristic of data. Examples of seasonality are: 1) Temperatures tend to be higher in the summer and lower in the winter; 2) There is more traffic on the roads during the rush hours.

Ramot et al. [30] introduced complex fuzzy sets as type-1 fuzzy sets extended by an additional phase term which permits them to represent fuzziness in time-series applications. A significant portion of this paper "... is dedicated to a discussion of the intuitive interpretation of complex-valued grades of membership." According to Dick [8], “... the proper role for a complex fuzzy set is a remarkably efficient representation of approximately periodic phenomena, and as the underlying mathematical foundation of regularity." Besides their ability to handle seasonality, another advantage of complex fuzzy sets is that they enable inferencing which takes account of constructive and destructive rule interference (Subsection III-B).

Since complex fuzzy sets are a fairly recent innovation, to date their applications are relatively few in number. They include the analysis of solar activity as measured by the recorded number of sunspots [30], signal processing [30], stock trading on the New York Stock Exchange [29], prediction of voter turnout in elections [29], multi periodic factor prediction [24] and multi-variate time-series forecasting [37].

Type-2 fuzzy sets [39], [40], [41] are an extension of type1 fuzzy sets in which the sets' membership grades are themselves type-1 fuzzy sets. They respond to a major shortcoming of type-1 fuzzy sets by offering a conceptual scheme within which the effects of uncertainties in fuzzy inferencing may be modelled and minimised [27, page 117]. Type-2 fuzzy logic is well-suited to reasoning under uncertainty [14], whereas complex fuzzy logic is pertinent to inferencing with seasonal data [29]. So far, these two sorts of fuzzy logic have been developed independently, but there is no reason why they may not be combined into type-2 complex fuzzy logic [12], able to deal with both uncertainty and seasonality. Combining these two paradigms would allow a more subtle and faithful treatment of data, leading to more precision in its analysis. There is every reason to suppose that this combined approach would outperform type-2 fuzzy logic and complex fuzzy logic [24], employed, as at present, in isolation. This would have the desirable effect of improving accuracy in data analysis.

Type-2 fuzzy sets take two forms, generalised, and the simpler interval. Interval type-2 fuzzy sets have increasingly been used in applications [15], [22], [3], [10], [19], [35], [17], [31] as they offer a more sophisticated model of uncertainty than their type-1 counterparts [23], whilst lacking the computational complexity of the generalised type-2 fuzzy set [27], [16]. 
Another form of fuzzy set is the interval-valued fuzzy set, which is regarded as equivalent to the interval type-2 fuzzy set [4, Definition 1, page 217]. This paper is concerned with the combination of interval-valued fuzzy sets and complex fuzzy sets to develop interval-valued complex fuzzy sets, an adaption of complex fuzzy sets such that the membership function assigns each point on the domain to an interval.

Through the Fuzzy Inferencing System (FIS), fuzzy logic operators are applied to linguistic rules. This paper is solely concerned with the Mamdani FIS in which a crisp numerical input passes through three stages: fuzzification, inferencing, and finally defuzzification. The output of inferencing is a fuzzy set known as the aggregated set. During the defuzzification stage the aggregated set is converted into a crisp number, the final result of the processing of the FIS. Figure 1 is a representation of a Mamdani type-1 FIS. In a Mamdani type-

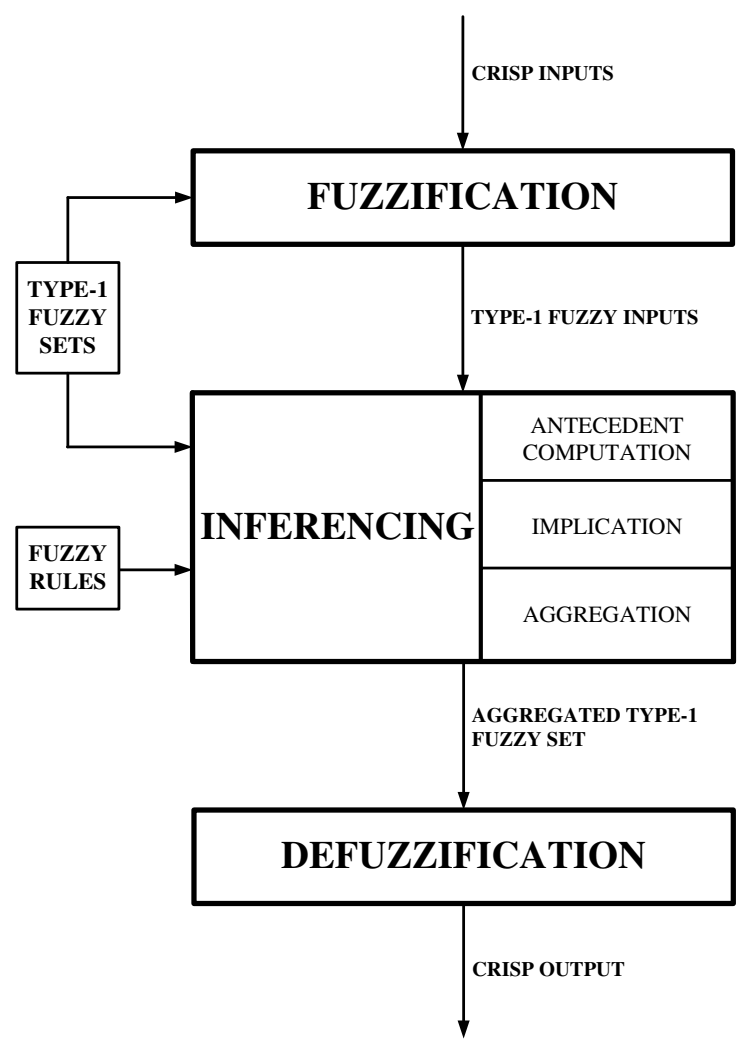

Fig. 1. Type-1 Mamdani FIS

2 FIS, type-1 defuzzification follows type-reduction as the second stage of type-2 defuzzification.

Interval type-2 FISs have been shown to be superior to their type-1 counterparts [26], [9], [1], evidence that it is reasonable to expect that interval-valued complex FISs will outperform complex FISs (Subsection IV-A).

Subramanian et al. [33], [34], in relation to classification problems, have proposed a complex-valued interval type-2 FIS (Figure 4), which is an interval type-2 FIS responsive to complex-valued inputs. This is not equivalent to the system proposed here, whose inputs conform to Definition ?? below.

In the next section, the interval-valued complex fuzzy set is defined. Following that, in Section III, the logical operations required for interval-valued complex fuzzy inferencing are developed. Section IV concludes the paper.

\section{InTERVAl-VAlued COMPlex Fuzzy Sets: DEFINITION}

\section{A. Type-1 Fuzzy Sets}

Since complex fuzzy sets and type- 2 fuzzy sets both derive from the basic type-1 fuzzy set, we begin by formally defining the type-1 fuzzy set.

Definition 1 (Type-1 Fuzzy Set). Let $X$ be a universe of discourse. A type-1 fuzzy set $A$ on $X$ is characterised by a membership function $\mu_{A}: X \rightarrow[0,1]$ and can be expressed as follows [38]:

$$
A=\left\{\left(x, \mu_{A}(x)\right) \mid \mu_{A}(x) \in[0,1] \forall x \in X\right\} .
$$

Note that the membership grades of $A$ are crisp, real numbers.

\section{B. Interval-Valued Fuzzy Sets}

Definition 2 (Interval-Valued Fuzzy Set [4, page 217]). An interval-valued fuzzy set $a$ on the universe $U \neq \emptyset$ is a mapping $A: U \rightarrow L([0,1])$, such that the membership degree of $u \in U$ is given by

$$
A(u)=[\underline{A}(u), \bar{A}(u)] \in L([0,1]),
$$

where $\underline{A}: U \rightarrow[0,1]$ and $\bar{A}: U \rightarrow[0,1]$ are mappings respectively defining the lower and upper of the membership interval $A(u)$.

\section{Type-2 Fuzzy Sets}

We shall now extend the type-1 fuzzy set definition to define the type-2 fuzzy set. In the following the notation $U=[0,1]$ will be used. Let $\tilde{P}(U)$ be the set of fuzzy sets in $U$. A type-2 fuzzy set $\tilde{A}$ in $X$ is a fuzzy set whose membership grades are themselves fuzzy [39], [40], [41]. This implies that $\mu_{\tilde{A}}(x)$ is a fuzzy set in $U$ for all $x$, i.e. $\mu_{\tilde{A}}: X \rightarrow \tilde{P}(U)$ and

$$
\tilde{A}=\left\{\left(x, \mu_{\tilde{A}}(x)\right) \mid \mu_{\tilde{A}}(x) \in \tilde{P}(U) \forall x \in X\right\} .
$$

It follows that $\forall x \in X \exists J_{x} \subseteq U$ such that $\mu_{\tilde{A}}(x): J_{x} \rightarrow U$. Applying (1), we obtain:

$$
\begin{aligned}
\mu_{\tilde{A}}(x)= & \left\{\left(u, \mu_{\tilde{A}}(x)(u)\right) \mid \mu_{\tilde{A}}(x)(u) \in U\right. \\
& \left.\forall u \in J_{x} \subseteq U\right\} .
\end{aligned}
$$

$X$ is called the primary domain and $J_{x}$ the primary membership of $x$ while $U$ is known as the secondary domain and $\mu_{\tilde{A}}(x)$ the secondary membership of $x$.

Putting (3) and (4) together we obtain

$$
\begin{gathered}
\tilde{A}=\left\{\left(x,\left(u, \mu_{\tilde{A}}(x)(u)\right)\right) \mid \mu_{\tilde{A}}(x)(u) \in U,\right. \\
\left.\forall x \in X \wedge \forall u \in J_{x} \subseteq U\right\} .
\end{gathered}
$$

This is the definition of the (generalised) type-2 fuzzy set (Figure 2(a)), an important property which is the footprint of uncertainty (Figure 2(b)). 
Definition 3 (Footprint Of Uncertainty). The Footprint Of Uncertainty (FOU) is the projection of the type-2 fuzzy set onto the $x-u$ plane.

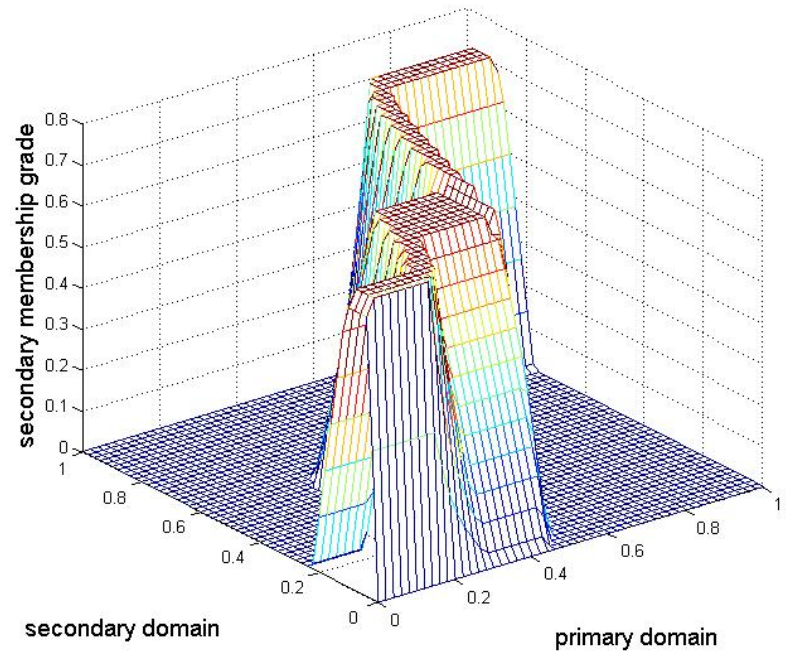

(a) 3-D representation

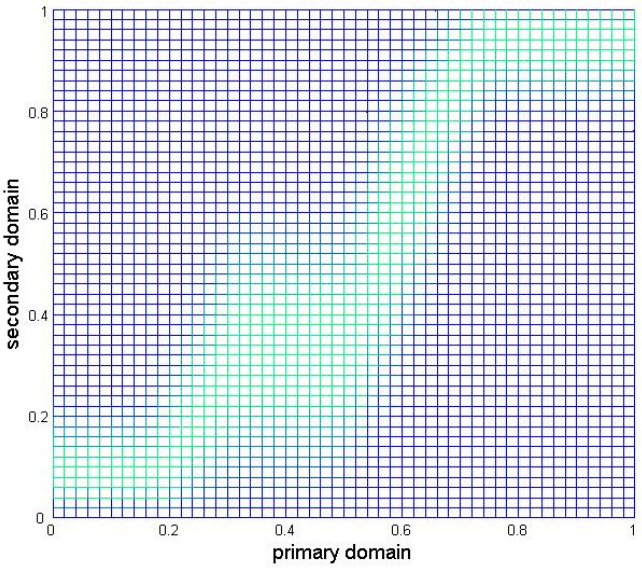

(b) FOU

Fig. 2. Aggregated type-2 fuzzy set created during the inference stage of a type-2 FIS coded in Matlab ${ }^{T M}$.

Definition 4 (Interval Type-2 Fuzzy Set). An interval type-2 fuzzy set is a type-2 fuzzy set whose secondary membership grades are all 1 .

In the interval case, Equation 5 reduces to:

$$
\tilde{A}=\left\{(x,(u, 1)), \quad \forall x \in X \wedge \forall u \in J_{x} \subseteq U\right\} .
$$

Since an interval type-2 fuzzy set is defined by its FOU, it is equivalent to an interval-valued fuzzy set.

\section{Complex Fuzzy Sets}

Complex fuzzy sets are type-1 fuzzy sets that have an additional phase component to the membership function. They are defined via polar co-ordinates.
Definition 5 (Complex Fuzzy Set [30, page 172]). "A complex fuzzy set $S$, defined on a universe of discourse $U$, is characterized by a membership function $\mu_{S}(x)$ that assigns any element $x \in U$ a complex-valued grade of membership in $S$. By definition, the values $\mu_{S}(x)$ may receive all lie within the unit circle in the complex plane, and are thus of the form $r_{S}(x) \cdot e^{j \omega_{S}(x)}$, where $j=\sqrt{-1}, r_{S}(x)$ and $\omega_{S}(x)$ are both real-valued, and $r_{s}(x) \in[0,1]$.

The complex fuzzy set $S$ may be represented as the set of ordered pairs

$$
S=\left(x, \mu_{S}(x)\right) \mid x \in U . "
$$

Figure 3 shows the sunspot observations of [2] displayed as a complex fuzzy set. This way of displaying time-series clearly shows the coiled or spiral effect engendered by the phase component.

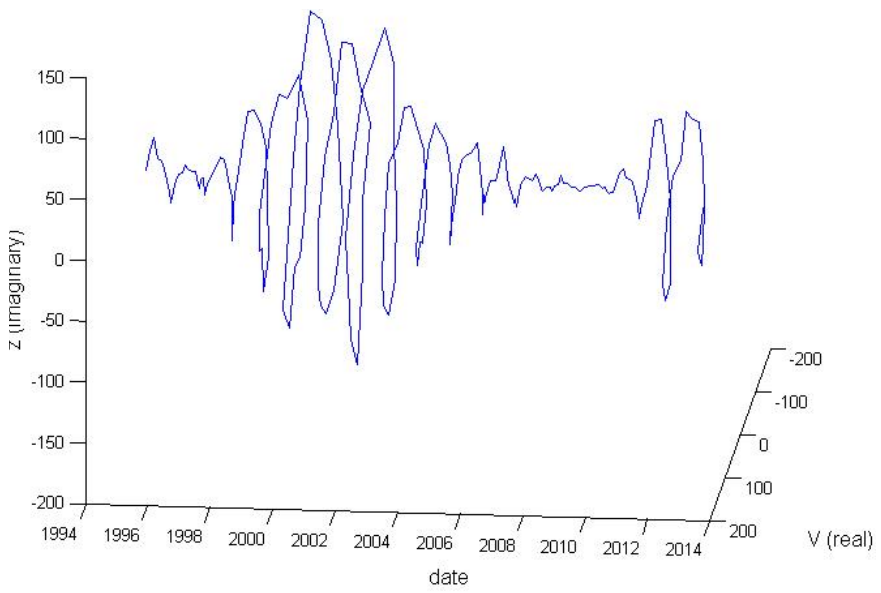

Fig. 3. Sunspot data [2] represented as a complex fuzzy set. The modulus is greater than 1 because the sunspot data has not been normalised.

\section{E. Interval-Valued Complex Fuzzy Sets}

The definition for the complex fuzzy set may be adapted so that the membership function assigns each point on the domain to an interval, giving the IVCFS. Before formally defining the IVCFS, we define concept of the dot product set.

Definition 6 (Dot Product Set). Given two sets $A$ and $B$ and an operator - such that given $a \in A$ and $b \in B$ the element $a \cdot b$ exists, the set $A \cdot B$ can be defined:

$$
A \cdot B=\{a \cdot b ; a \in A \wedge b \in B\} .
$$

Definition 7 (Interval-Valued Complex Fuzzy Set). An interval-valued complex fuzzy set over a universe of discourse $U$ is defined by a membership function

$$
\begin{gathered}
\mu_{\bar{S}}: U \rightarrow I^{[0,1]} \cdot \dot{D} \\
\mu_{\bar{S}}(x)=r_{\bar{S}}(x) \cdot e^{j \omega_{\bar{S}}(x)}
\end{gathered}
$$

where $I^{[0,1]}$ is the set of all closed subintervals of $[0,1], \dot{D}$ is the frontier or boundary set of the unit disc $D \subset \mathbb{R} \times \mathbb{R}$, $r_{\bar{S}}(x) \in I^{[0,1]}$ is the interval-valued membership magnitude, 
$j=\sqrt{-1}$, and $\omega_{\bar{S}}(x)$ is the membership phase. Each element of the universe of discourse $U$ is thus associated with a set of complex numbers with moduli in a subinterval of [0,1] representing the range of possible truth-values associated with that element's membership in the complex fuzzy set, and a real-valued phase.

Graphically, the 3-D interval-valued complex fuzzy set $\bar{S}$ corresponds to a band of points of global variable width and constant local phase within the complex plane unit circle. The interval-valued complex fuzzy set is strictly speaking not a type-2 fuzzy set; the third dimension arises from the phase component of the type-1 membership function. Since it is not a type-2 fuzzy set, it does not possess an FOU.

The definition of the interval-valued complex fuzzy set may be extended further to give that of the 4-D generalised type-2 complex fuzzy set, discussion of which is beyond the scope of this paper. Figure 4 indicates how the different forms of fuzzy logic relate to each other.

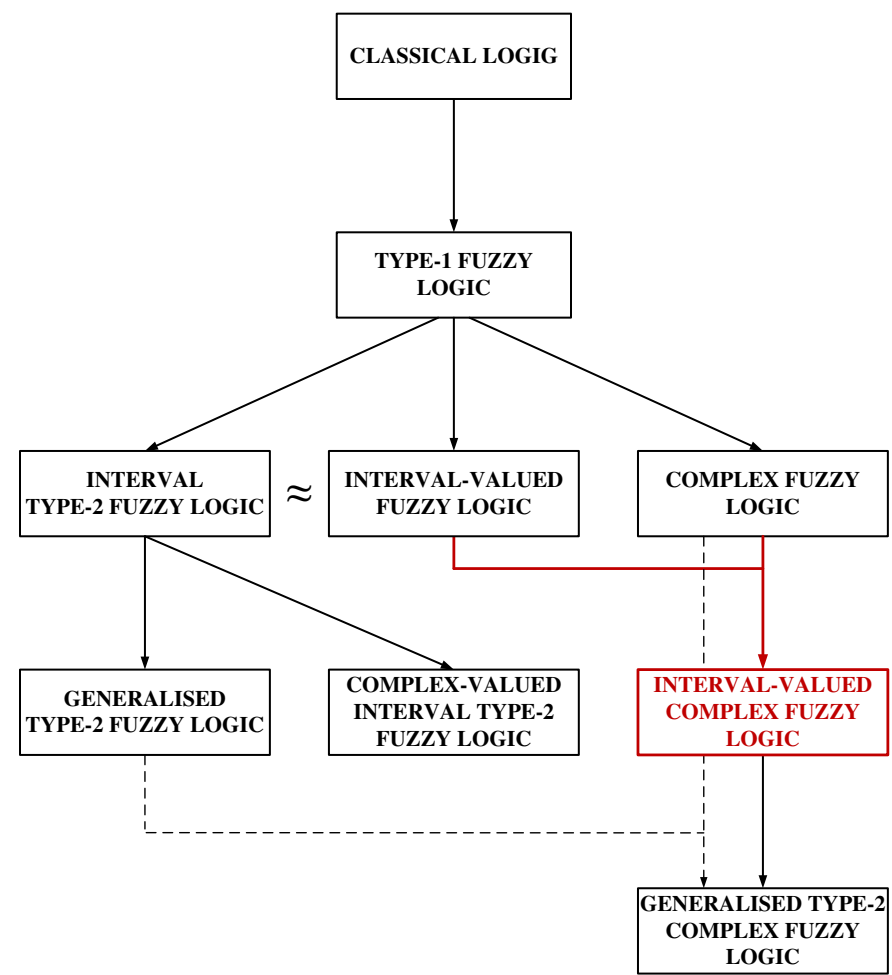

Fig. 4. Relationship between classical logic and various forms of fuzzy logic. Interval-valued complex fuzzy logic, is indicated in red.

\section{INTERVAL-VALUED COMPLEX FIS: Operations}

An interval-valued complex FIS is a hybrid of a complex FIS and an interval-valued FIS. An interval-valued FIS is equivalent to an interval type-2 FIS. It would be unsurprising if the interval-valued complex FIS were to inherit properties from both the complex FIS and the interval type-2 FIS. Figure
5 shows the various stages of the interval-valued complex FIS. Each stage is discussed in turn below.

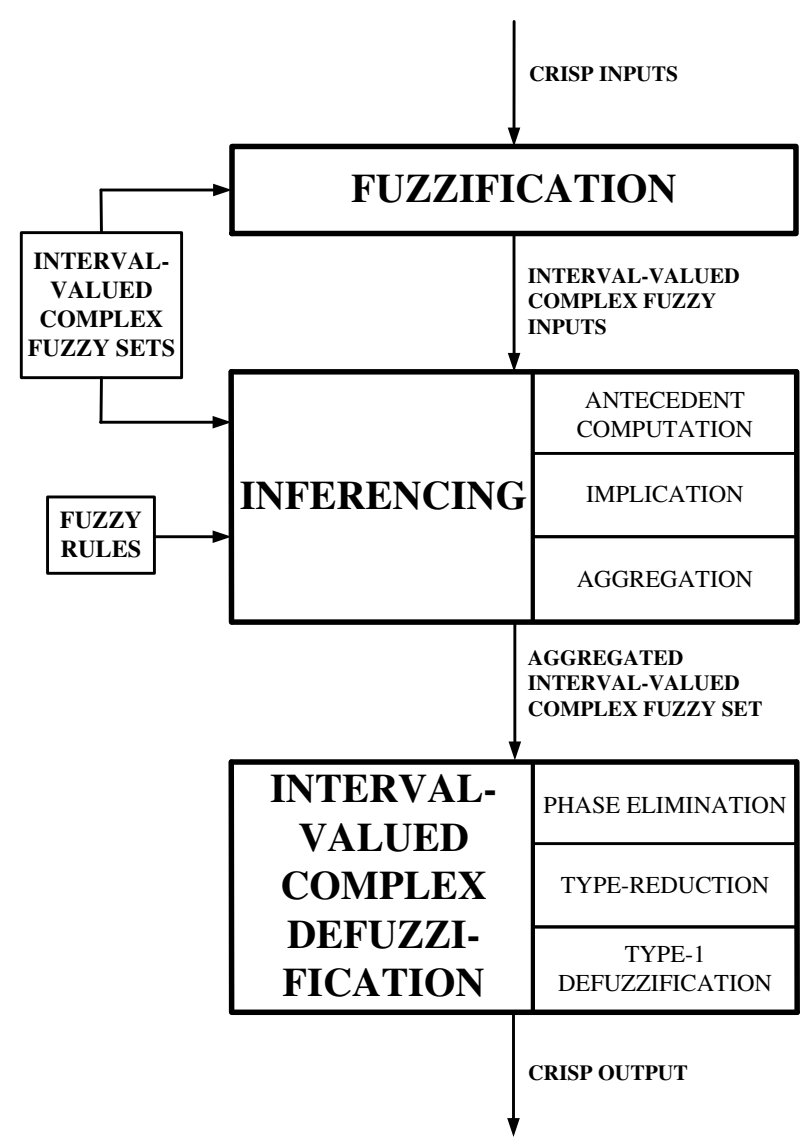

Fig. 5. The interval-valued complex Mamdani FIS.

\section{A. Fuzzification, Antecedent Combination, and Implication}

1) Complex Union and Intersection: The fuzzification stage, and the antecedent combination and implication substages of the inferencing stage are driven by union and intersection operations in the type-1 case. Ramot et al. [29, page 452] extend these operations to complex fuzzy sets. Union is defined thus:

"Let $\mu_{A}(x)=r_{A}(x) \cdot e^{j \omega_{A}(x)}$ and $\mu_{B}(x)=r_{B}(x)$. $e^{j \omega_{B}(x)}$. Then, the membership function of $A \cup B$ is given by

$$
\mu_{A \cup B}(x)=\left[r_{A}(x) \oplus r_{A}(x)\right] \cdot e^{j \omega_{A \cup B}(x)}
$$

where $\oplus$ represents a t-conorm function."

Suggested techniques for calculating $A \cup B$ are [29, page 452]:

1) Sum: $\omega_{A \cup B}=\omega_{A}+\omega_{B}$;

2) Maximum: $\omega_{A \cup B}=\max \left(\omega_{A}, \omega_{B}\right)$;

3) Minimum: $\omega_{A \cup B}=\min \left(\omega_{A}, \omega_{B}\right)$;

4) Winner Takes All: $\omega_{A \cup B}=\left\{\begin{array}{l}\omega_{A}, r_{A}>r_{B} \\ \omega_{B}, r_{B}>r_{A}\end{array}\right.$ 
Options 2, 3 and 4 have the 'intuitively appealing property' of rotational invariance [8]. Intersection is defined analogously to union (using a t-norm instead of a t-conorm). Suggested techniques for calculating $A \cap B$ are the same as those for calculating $A \cup B$.

2) Interval-Valued Complex Join and Meet: We now extend the definitions of complex union and intersection to intervalvalued complex join and meet. Considering join first, the tconorm in Equation 7 for complex union may be replaced by the interval type- 2 join operation [25]. Of the techniques suggested for finding $A \cup B$, the first three are independent of the modulus, so may be employed for interval-valued complex join without adaptation. The fourth technique includes moduli among the parameters. These are crisp numbers, as opposed to to intervals. The intervals in the interval-valued complex membership functions need to be reduced to crisp numbers in order for the fourth technique to be applicable, achieved easily by taking the mid-point of the interval as a representative value, though any method of ranking interval numbers [7] would be a valid alternative. Then the definitions of complex union may be applied, with the moduli replaced by the interval midpoints. The meet operation may be defined analogously to the join operation.

\section{B. Aggregation}

The aggregation stage requires an aggregation operator. Leaving data seasonality aside, a distinctive advantage of complex fuzzy inferencing over non-complex type-1 fuzzy inferencing is that it provides for rule interference [29] [8, page 406]. Rule interference occurs when rule outputs interact in a manner that is dependant upon their relative phases, and can be either constructive or destructive. It is enabled at the aggregation stage of the complex FIS by the vector aggregation operator. According to Ramot et al.,

"Vector aggregation is in essence a vector sum of its arguments. Therefore, the result of vector aggregation is strongly dependent on the relative phase of its arguments. For example, if all of the arguments are in phase, the amplitude of the sum is maximized. If, however, the arguments are not in phase, the result of the sum may be a grade of membership whose amplitude is smaller than that of its separate arguments. As amplitude is a parameter of consequence in the defuzzification stage, relative phase between inference rules is of great importance. Thus, a form of interaction between rules in a CFLS is defined ..." [29, page 457]

Another candidate for aggregation operator is the intervalvalued complex join operator proposed above; this approach, however, does not facilitate rule interference.

\section{Phase Elimination}

In Figure 3, the coiled effect induced in a complex fuzzy set by assigning a phase component to a type- 1 fuzzy set is clearly visible. In the case of an interval-valued complex fuzzy set, instead of a spiral line, there is a spiral band. The first stage in the defuzzification of an interval-valued complex fuzzy set consists of uncoiling, or unwinding, the spiral. In this way the phase component is eliminated, so transforming an intervalvalued complex fuzzy set to an interval-valued fuzzy set.

\section{Type-Reduction}

For interval-valued fuzzy sets (which are equivalent to interval type-2 fuzzy sets), the leading type-reduction methods are the Karnik-Mendel Iterative Procedure [18], the Nie-Tan Method [28], and the Greenfield-Chiclana Collapsing Defuzzifier (GCCD) [13]. They are contrasted in [11]; the GCCD is shown to outperform the other methods in terms of accuracy, making it the method of choice for interval type-reduction.

\section{E. Type-1 Defuzzification}

For type-1 defuzzification, there are various strategies [21]. Most frequently employed is the centroid technique [6], [32].

\section{CONCLUSION}

This investigation introduces and develops the theory of a new form of fuzzy logic, interval-valued complex fuzzy logic, whose particular application is to reasoning under both uncertainty and seasonality. The interval-valued complex fuzzy set was defined, after which the logical operations comprising the interval-valued complex FIS were presented.

\section{A. Further Work}

1) Rule Interference: Rule interference is a significant characteristic of the complex FIS (Subsection III-B). In Layer 4 of the ANCFIS architecture proposed by Chen et al. [5], rule interference in aggregation is achieved by calculating the dot product of the complex firing strengths. This calculation simultaneously and additionally accomplishes both phase elimination and type reduction. Further investigation is needed in order to extend this approach to interval-valued fuzzy inferencing.

In the context of the complex FIS, to enable rule interference, Ramot et al. proposed the vector aggregation operator [29, page 453]. This operator, as well as the t-norm and tconorm operators driving fuzzification, antecedent computation, and implication, is applicable to crisp inputs. However, these operators may be extended to the situation where the inputs are type-1 fuzzy sets via the Extension Principle [39] and the Representation Theorem [27]. The type-1 fuzzy set generated may be regarded as an interval-valued fuzzy set, which is a fuzzy set with just one $\alpha$-cut [20, pages 16-18], the 1- $\alpha$-cut. For an example of this strategy, please refer to [36]. Further development of this approach would be desirable.

2) Prototype Interval-Valued Complex FIS: The development of a prototype interval-valued complex FIS would be welcome. This could be any application with uncertain inputs where either seasonality or rule interference is a consideration. Its performance could be contrasted with a crisp complex FIS and a conventional interval type-2 FIS.

3) Generalised Type-2 Complex FIS: Closely connected with the work reported in this paper would be the development of generalised type-2 complex fuzzy inferencing. 


\section{REFERENCES}

[1] Jabran Hussain Aladi, Christian Wagner, and Jonathan M. Garibaldi. Type-1 or Interval Type-2 Fuzzy Logic Systems - On the Relationship of the Amount of Uncertainty and FOU Size. In Proceedings of FUZZIEEE 2014, pages 2360 - 2367, Beijing, China, 2014.

[2] Australian Government Bureau of Meteorology: Radio and Space Weather Services. Monthly Sunspot Numbers, 2013. http://www.ips. gov.au/Solar/1/6 [Online; accessed 21-February-2013].

[3] Nora Boumella, Karim Djouani, and Mohamed Boulemden. On an Interval Type-2 TSK FLS A1-C1 Consequent Parameters Tuning. In Proceedings of the IEEE Symposium on Advances in Type-2 Fuzzy Logic Systems 2011, Paris, April 2011.

[4] Humberto Bustince. Interval-valued fuzzy sets in soft computing. International Journal of Computational Intelligence Systems, 3(2):215 $-222,2010$.

[5] Zhifei Chen, Sara Aghakhani, James Man, and Scott Dick. ANCFIS: A Neurofuzzy Architecture Employing Complex Fuzzy Sets. IEEE Transactions on Fuzzy Systems, 19(2):305 - 322, 2011.

[6] Earl Cox. Fuzzy fundamentals. IEEE Spectrum, 29(10):58 - 61, October 1992.

[7] Joaquín Derrac, Francisco Chiclana, Salvador García, and Francisco Herrera. Evolutionary fuzzy k-nearest neighbors algorithm using intervalvalued fuzzy sets. Information Sciences, 329:144 - 163, 2016.

[8] Scott Dick. Toward Complex Fuzzy Logic. IEEE Transactions on Fuzzy Systems, 13(3):405-414, June 2005. DOI: http://dx.doi.org/10.1109/TFUZZ.2004.839669.

[9] Umar Farooq and Jhen-Fong Gu. Design and Comparison of Type-1 and Interval Type-2 Fuzzy Gain Scheduling Controllers for Ball and Beam System. In Proceedings of the International Conference on Information \& Communication Technologies (ICICT) 2013, pages 1-7, 2013.

[10] Mikel Galar, Edurne Barrenechea, Javier Fernandez, Humberto Bustince, and Gleb Beliakov. Representing Images by Means of Interval-Valued Fuzzy Sets. Application to Stereo Matching. In Proceedings of the IEEE Symposium on Advances in Type-2 Fuzzy Logic Systems 2011, Paris, April 2011.

[11] Sarah Greenfield and Francisco Chiclana. Accuracy and Complexity Evaluation of Defuzzification Strategies for The Discretised Interval Type-2 Fuzzy Set. International Journal of Approximate Reasoning, 54(8):1013 - 1033, October 2013. DOI: http://dx.doi.org/10.1016/j.jjar.2013.04.013.

[12] Sarah Greenfield and Francisco Chiclana. Fuzzy in 3D: Two Contrasting Paradigms. Archives for the Philosophy and History of Soft Computing, 2, 2015. http://www.aphsc.org/index.php/aphsc/article/view/24 [Online; accessed 20-January-2016].

[13] Sarah Greenfield, Francisco Chiclana, Simon Coupland, and Robert I. John. The Collapsing Method of Defuzzification for Discretised Interval Type-2 Fuzzy Sets. Information Sciences, 179(13):2055-2069, June 2009. DOI: http://dx.doi.org/10.1016/j.ins.2008.07.011.

[14] Sarah Greenfield and Robert I. John. The Uncertainty Associated with a Type-2 Fuzzy Set. In Rudolf Seising (editor) Views on Fuzzy Sets and Systems from Different Perspectives, in 'Studies in Fuzziness and Soft Computing', series editor Janusz Kacprzyk, volume 243, pages 471483. Springer-Verlag, 2009. DOI: http://dx.doi.org/10.1007/978-3-54093802-6_23.

[15] Hani Hagras and Christian Wagner. Introduction to Interval Type2 Fuzzy Logic Controllers - Towards Better Uncertainty Handling in Real World Applications. IEEE Systems, Man and Cybernetics eNewsletter, 2009. Issue 27.

[16] Robert I. John and Simon Coupland. Type-2 Fuzzy Logic: A Historical View. IEEE Computational Intelligence Magazine, 2(1):57 - 62, February 2007. DOI: 10.1109/MCI.2007.357194.

[17] Aranzazu Jurio, Daniel Paternain, Carlos Lopez-Molina, Humberto Bustince, Radko Mesiar, and Gleb Beliakov. A Construction Method of Interval-Valued Fuzzy Sets for Image Processing. In Proceedings of the IEEE Symposium on Advances in Type-2 Fuzzy Logic Systems 2011, Paris, April 2011.

[18] Nilesh N. Karnik and Jerry M. Mendel. Centroid of a Type-2 Fuzzy Set. Information Sciences, 132:195 - 220, 2001

[19] Erdal Kayacan, Ozkan Cigdem, and Okyay Kaynak. On Novel Training Method Based on Variable Structure Systems Approach for Interval Type-2 Fuzzy Neural Networks. In Proceedings of the IEEE Symposium on Advances in Type-2 Fuzzy Logic Systems 2011, Paris, April 2011.
[20] George J. Klir and Tina A. Folger. Fuzzy Sets, Uncertainty, and Information. Prentice-Hall International, 1992

[21] Werner Van Leekwijck and Etienne E. Kerre. Defuzzification: Criteria and Classification. Fuzzy Sets and Systems, 108:159 - 178, 1999. DOI: 10.1016/j.fss.2008.06.018

[22] Leonardo Leottau and Miguel Melgarejo. Implementing an Interval Type-2 Fuzzy Processor onto a DSC 56F8013. In Proceedings of FUZZIEEE 2010, pages 1939-1942, Barcelona, Spain, 2010.

[23] Ondrej Linda and Milos Manic. Uncertainty Modelling for Interval Type-2 Fuzzy Logic Systems Based on Sensor Characteristics. In Proceedings of the IEEE Symposium on Advances in Type-2 Fuzzy Logic Systems 2011, Paris, April 2011.

[24] Jun Ma, Guangquan Zhang, and Jie Lu. A Method for Multiple Periodic Factor Prediction Problems Using Complex Fuzzy Sets. IEEE Transactions on Fuzzy Systems, 20(1):32 - 45, 2012. DOI: http://dx.doi.org/10.1109/TFUZZ.2011.2164084

[25] Jerry M. Mendel. Uncertain Rule-Based Fuzzy Logic Systems: Introduction and New Directions. Prentice-Hall PTR, 2001.

[26] Jerry M. Mendel. A Quantitative Comparison of Interval Type-2 and Type-1 Fuzzy Logic Systems: First Results. In Proceedings of FUZZIEEE 2010, pages $1-8$, Barcelona, Spain, 2010.

[27] Jerry M. Mendel and Robert I. John. Type-2 fuzzy sets made simple. IEEE Transactions on Fuzzy Systems, 10(2):117 - 127, 2002. DOI: http://dx.doi.org/10.1109/91.995115.

[28] Maowen Nie and Woei Wan Tan. Towards an Efficient Type-Reduction Method for Interval Type-2 Fuzzy Logic Systems. In Proceedings of FUZZ-IEEE 2008, pages 1425 - 1432, Hong Kong, June 2008.

[29] Daniel Ramot, Menahem Friedman, Gideon Langholz, and Abraham Kandel. Complex Fuzzy Logic. IEEE Transactions on Fuzzy Systems, 11(4):450 - 461, 2003. DOI: http://dx.doi.org/10.1109/TFUZZ.2003.814832.

[30] Daniel Ramot, Ron Milo, Menahem Friedman, and Abraham Kandel. Complex Fuzzy Sets. IEEE Transactions on Fuzzy Systems, 10(2):171 - 186, 2002. DOI: http://dx.doi.org/10.1109/91.995119.

[31] José A. Sanz, Alberto Fernández, Humberto Bustince, and Francisco Herrera. A Genetic Tuning to Improve the Performance of Fuzzy RuleBased Classification Systems with Interval-Valued Fuzzy Sets: Degree of Ignorance and Lateral Position. International Journal of Approximate Reasoning, 52:751-766, 2011.

[32] Gilberto C. D. Sousa and Bimal K. Bose. A Fuzzy Set Theory Based Control of a Phase-Controlled Converter DC Machine Drive. IEEE Transactions on Industry Applications, 30(1):34 - 44, January/February 1994.

[33] Kartick Subramanian, Ramasamy Savitha, and Sundaram Suresh. A Metacognitive Complex-Valued Interval Type-2 Fuzzy Inference System. IEEE Transactions on Neural Networks and Learning Systems, 25(9): 1659.

[34] Kartick Subramanian and Sundaram Suresh. Evolving Complex-Valued Interval Type-2 Fuzzy Inference System. In Proceedings of the IEEE International Conference on Fuzzy Systems (FUZZ-IEEE) 2015, pages $1-6,2015$.

[35] Arturo Tellez-Velazquez, Heron Molina-Lozano, Marco A. MorenoArmendariz, Elsa Rubio-Espino, Luis A. Villa-Vargas, and Ildar Batyrshin. Parametric Type-2 Fuzzy Control Design for the Ball and Plate System. In Proceedings of the IEEE Symposium on Advances in Type-2 Fuzzy Logic Systems 2011, Paris, April 2011.

[36] Jian Wu and Francisco Chiclana. Multiplicative consistency of intuitionistic reciprocal preference relations and its application to missing values estimation and consensus building. Knowledge-Based Systems, 71:187 - 200, 2014.

[37] Omolbanin Yazdanbakhsh and Scott Dick. Multi-Variate Timeseries Forecasting Using Complex Fuzzy Logic. In Proceedings of NAFIPS 2015, pages $1-6$, Redmond, Washington, 2015.

[38] Lotfi A. Zadeh. Fuzzy Sets. Information and Control, 8:338 - 353, 1965.

[39] Lotfi A. Zadeh. The Concept of a Linguistic Variable and its Application to Approximate Reasoning. Information Sciences, 8:199 - 249, 1975.

[40] Lotfi A. Zadeh. The Concept of a Linguistic Variable and its Application to Approximate Reasoning - II. Information Sciences, 8:301 - 357, 1975.

[41] Lotfi A. Zadeh. The Concept of a Linguistic Variable and its Application to Approximate Reasoning - III. Information Sciences, 9:43 - 80, 1975. 